\title{
Iris Recognition Method Based On Natural Open Eyes
}

\author{
Latha B. M*, Kumar L. \\ Department of Electronics, GM Institute of Technology, Davangere-577006, Karnataka, India
}

\begin{tabular}{|c|c|}
\hline Abstract & Article Information \\
\hline \multirow{5}{*}{$\begin{array}{l}\text { The non-intrusive property of iris recognition leads to several problems to the } \\
\text { images of natural-open eyes and it is hard to increase the accuracy of iris } \\
\text { recognition because of these problems. In order to ensure the non-intrusive } \\
\text { property as well as achieve an iris recognition which has high accuracy } \\
\text { simultaneously, this paper presents a novel iris recognition method based on the } \\
\text { natural-open eyes. Firstly, makes pre-process to iris image, ensures the effective } \\
\text { iris area adaptively. Secondly, finds all iris feature points by directional } \\
\text { information, length information, width information of texture, the neighbouring } \\
\text { gray information and relativity in the effective iris area. Thirdly, makes codes to } \\
\text { feature points and figures the iris pattern by iris codes. Finally, sorts the different } \\
\text { iris patterns by auto accommodated pattern matching method and gives the } \\
\text { recognition results. Many experiments show the recognition rates of this method } \\
\text { can reach } 99.687 \% \text { that can meet the demand of iris recognition. }\end{array}$} & $\begin{array}{l}\text { Article History: } \\
\text { Received : }: 05-01-2012 \\
\text { Revised : 18-02-2012 } \\
\text { Accepted : } 22-02-2012\end{array}$ \\
\hline & Keywords: \\
\hline & $\begin{array}{l}\text { Iris recognition } \\
\text { Natural-open } \\
\text { Adaptive }\end{array}$ \\
\hline & ${ }^{\star}$ Corresponding Author: \\
\hline & \\
\hline
\end{tabular}

\section{INTRODUCTION}

As an important and distinct characteristic for status, the iris has many advantages such as uniqueness, stability, may gathering and so on. Non-contacting biometrics is the inevitable trend for the research and the development of status identification. The error rate of iris recognition is the lowest in all the biometrics according to the statistics. Daugman presented an algorithm that needs to process the two dimensional information of the texture, and increases feature extraction time; Wildes used the Gauss-Laplace filter to decompose the iris image under the different resolution, and carried on the correlation comparison for the corresponding images, the computation is huge; Boles and Boashash proposed a novel iris recognition algorithm based on zero crossing detection of the wavelet transform, this method has only obtained the limited results in the small samples, and this algorithm is sensitive to the grey value changes, thus recognition rate is lower.
This paper presents a novel iris recognition method based on the natural-open eyes. Firstly, it makes preprocess to iris image, ensures the effective iris area adaptively. Secondly, it finds all iris feature points by directional information, length information, width information of texture, the neighbouring gray information and relativity in the effective iris area. Thirdly, it makes codes to feature points and figures the iris pattern by iris codes. Finally, it sorts the different iris patterns by auto accommodated pattern matching method and gives the recognition results.

\section{Image Pre-processing}

Usually an iris image impossibly contains the iris merely; there are also other parts of the eye such as the eyelid, the eyelash. This point may be seen clearly from Figure 1. The interior boundary of iris can change, and make the texture of iris distort. In order to realize exactly matching, it must eliminate these factors through the image pre-processing. Iris image pre- 
processing includes iris localization, eyelid fitting, eyelash detection and normalization.

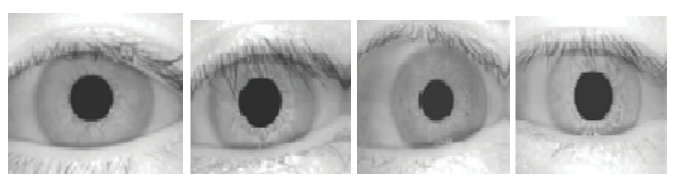

Figure 1. Iris images.

\section{Iris localization}

Firstly, it finds the sketchy pupil center through the gray projection and the pupil center detection operator; Secondly, finds four iris inner boundary points by the direction edge detection operator and the voting mechanism beginning from the sketchy pupil center, and locates the iris inner boundary according to these four points; Finally finds four iris outer boundary points by the direction edge detection operator and the voting mechanism beginning from the center of pupil, and locates the iris outer boundary according to these four points. Localization accuracy rate of this method is high, the speed is quick. The detail contents can be seen from literature.

\section{Fitting Lower Lid}

Firstly it uses canny operator to extraction edge information of iris image, then uses the parabolic equation (1) to fit the lower eyelid:

$$
y=a(x-b)^{2}+c
$$

$a$ is the parabola curvature; b, c are the horizontal and vertical coordinates of parabola apex respectively. Through establishing different $a, b$, and $c$ it may fit the lower eyelid well, thus it can eliminates influence of the lower eyelid for the effective iris region. It finds the parabola apex. The resultant images of Figure 1 are shown in Figure 2.
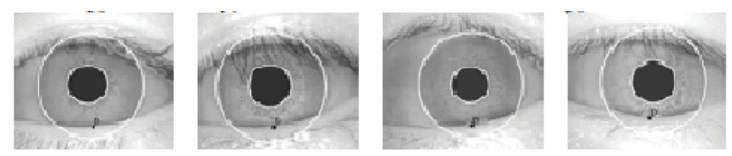

Figure 2. The resultant images of iris location and fitting the contour of the lower eyelid.

\section{Eyelash Detection}

Firstly, it makes sure the search area. The parameters of inner boundary and outer boundary are $\left(x_{p}, y_{p}, r_{p}\right)$ and $\left(x_{i}, y_{i}, r_{i}\right)$ respectively. It chooses two rectangles of the left and right sides of the pupil as the possible area covered by the eyelash. The four vertexes of right area are

$$
\begin{aligned}
& \left(x_{p}, y_{i}-r_{i}\right),\left(\text { lwidth }-1, y_{i}-r_{i}\right), \\
& \left(x_{p}, y_{i}+r_{i}\right),\left(\text { lwidth }-1, y_{i}+r_{i}\right) .
\end{aligned}
$$

The I width is the width of the image. The four vertexes of the left area are

$$
\begin{aligned}
& \left(0, y_{i}-r_{i}\right),\left(x_{p}, y_{i}-r_{i}\right), \\
& \left(0, y_{i}+r_{i}\right),\left(x_{p}, y_{i}+r_{i}\right) .
\end{aligned}
$$

Because the gray of eyelash is low, so a template of detection eyelash is designed, its shape is shown in Figure 3. Each sub-template $\mathrm{X} 1, \mathrm{X}, \mathrm{X} 2$ may compose by a single pixel or multiple pixels. If a sub-template is composed by a single pixel, this pixel corresponds the center of the sub template; if a sub-template is composed by $\mathrm{N}(\mathrm{N}>=2)$ pixels $\{\mathrm{a} 1, \mathrm{a} 2, \ldots \mathrm{aN}\}$, the center pixel locates at $\mathrm{n}=$ ceil( $\mathrm{N} / 2)$ and $a_{n}$ is the center pixel of the corresponding sub-template. It looks the center of $X$ as the current point and sums the gray difference with $\mathrm{X} 1, \mathrm{X} 2$ respectively, if two difference is less than 0 , the current point is taken as the candidate eyelash point. It searches in two rectangles and finds all candidates eyelash points according with conditions. Then it carries on thinning to the image that can ensure the directions, curvature and connectivity of eyelash, show the shape of eyelash well and delete some non-eyelash area. The gray of all candidate eyelash points are set 0 , the gray of other points are set 255 , so it gets the binary eyelash image.

\begin{tabular}{|c|c|c|}
\hline $\mathrm{X} 1$ & $\mathrm{X}$ & $\mathrm{X} 2$ \\
\hline
\end{tabular}

Figure 3. Eyelash Detection Template.

The found candidate eyelash points include true eyelash and false eyelash, so it needs to eliminate these false eye lashes. It includes two steps:(1) because eyelash mainly tends to vertical direction and has certain length, it can use the template shown in Figure 4. According to the length information and direction information of the eyelash it eliminates some false eyelash points. In Figure 4 point $A$ is the current candidate eyelash point, if one of these points $B L$ locating at the next line of point $A$ is candidate eyelash point, the corresponding length of eyelash adds 1 . It makes recursion for this operation until it reaches the bottom of eyelash. If the length of the curve is longer than $\mathrm{T}$, it is regarded as eyelash and kept, otherwise the curve is eliminated. Because the beginning point of eyelash is located near upper eyelid, the beginning point of the kept curve does not 
locate near the upper eyelid, the curve is eliminated too.

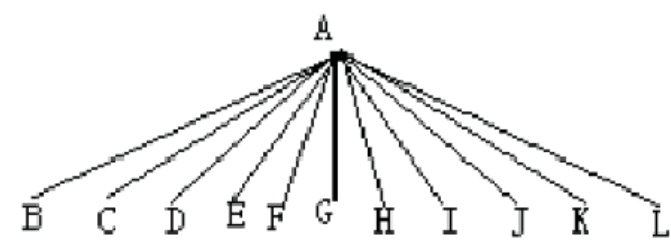

Figure 4. Length of eyelash detection template.

Finally it finds the end point of each kept eyelash curve, connects the center of the pupil and these end points and calculates the slope of each connecting lines, the formula of the slope is

$$
\frac{\left(y_{\text {daun }}-y_{p}\right)}{\left(x_{\text {daun }}-x_{p}\right)}
$$

in this formula $\left(x_{\text {dau } n} y_{\text {dau } n}\right)$ and $\left(x_{p}, y_{p}\right)$ express the coordinates of any end point and the center of pupil respectively. It retains the connecting line which locates at the left of the center of pupil with the minimum slope and the right of the center of pupil with maximum slope respectively. The above part of two connection lines and iris outer boundary compose fanshaped area, it removes the fan-shaped area, the area composing by the iris outer boundary and the parabola fitting lower eyelid, the rest area of the iris part is the effective area after removing noise. Figure 4 is the resultant image after removing noise of four images. The center points of two white small blocks express a point locating at the above two connecting lines respectively.

\section{Iris normalization}

After locating the iris, it cannot carry on the code for the locating iris image immediately, and should carry on the calibration firstly. Therefore it should adjust each primitive image to the same size and corresponding position through normalization. This article used the polar coordinate transform to carry on the normalization, because the inner and outer circles are not concentric, this kind of transform is not concentric. During the experiments, beginning from the upper vertical radius of pupil center it unwrapped the ring like iris to rectangular iris of $512 \times 64$ according to the counter clockwise. Because the rectangular coordinates obtaining from the polar coordinate are impossible integers during unwrapping, the gray values of pixels after unwrapping are computed by the bilinear interpolation. This kind of processing made the iris image have the scale-invariability and translation-invariability, rotation distort carried on the compensation in the following match processing. In the normalized image, the row direction coordinate of point $D$ which confirmed in section $B$ is $r D$. In Figure 1 four images normalized result as shown in Figure 5. The white rectangular area is the effective iris area. The width along vertical direction of the rectangle is $\mathrm{rD}$., the vertical line of the left part of rectangle corresponds the connecting line locating at the left of the center of pupil with the minimum slope confirmed in section $\mathrm{C}$ and the horizontal coordinate of each point in the line is $x_{l}$, the vertical line of the right part of rectangle corresponds the connecting line locating at the right of the center of pupil with the maximum slope confirmed in section $\mathrm{C}$ and the horizontal coordinate of each point in the line is $x_{r}$.
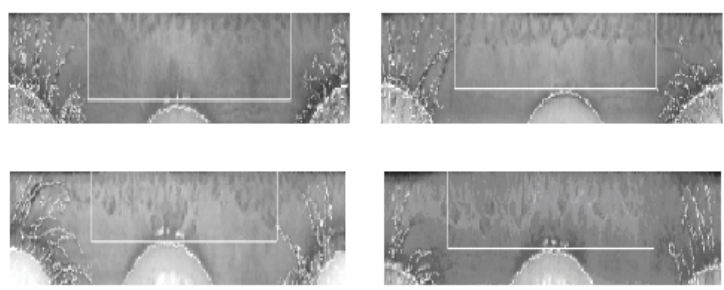

Figure 5. Processing image of pre-processing.

\section{Feature extraction and Code}

An iris image contains much detail texture, the texture is composed by many shape blocks such as strip and speckle, the gray differences are big and distribute unevenly, these blocks with irregular shape can be as distinguish characteristics for iris recognition. Firstly we need to determine the collective and effective coding region of the entering iris and the registering iris, this region does not contain noise such as eyelash, eyelid and facula. We suppose vertical coordinates of $D$ point of the entering iris and the registering iris in the normalized image are $r_{D}$ Enroll, $r_{D}$ Register respectively, and determine the smaller value as $r_{D}$ match between two values $x_{l}, x_{r}$ of the entering iris and the registering iris in the normalized image are $x_{l}$ Enroll $x_{r}$ Enroll, $x_{l}$ Register and $x_{r}$ register

respectively. Determine the bigger value as $x_{l}$ match between $x_{l}$ Enroll and $x_{l}$ Registe, and determine the smaller value as $x_{r}$ match $x_{r}$ Enroll and $x_{r}$ Register . So we determine collective and effective texture region of the entering iris and registering iris. 
Considering the block characteristics of iris texture, it first makes sub-block for the image, the size of the block is $M^{*} N(M$ and $N$ are integers) and ensures not overlap between each block. The number of block is (ceil $\left(\left(x_{r}\right.\right.$ match $-x_{l}$ match $) /$

$N)) X\left(\operatorname{ceil}\left(r_{D}\right.\right.$ match $\left.\left./ M\right)\right)$ in the collective and effective area of entering iris and registering iris the horizontal number of block is Hnum= $\left(\operatorname{ceil}\left(\left(x_{r}\right.\right.\right.$ match $-x_{l}$ match $\left.\left.) / N\right)\right)$ and the vertical number of block is Vnum $=\left(\operatorname{ceil}\left(r_{D}\right.\right.$ match $\left.\left./ M\right)\right)$.

In order to realize the compression code, it accumulates all the gray values in each block, the average of this accumulation is the gray value of the center point. During the feature extraction, it makes code by taking the center point of each block image as the basic feature point. This code method plays well in the compression, what is more, this cannot lose feature points.

In the collective and effective area of iris image, considering the texture characteristics which are the strength of the edge and direction information of texture it takes the basic feature point as the center point and considers the eight neighborhood of each center point, these eight points correspond to the four directional texture of the center point such as $45^{\circ}, 90^{\circ}, 135^{\circ}$ and $180^{\circ}$. Each direction corresponds to two adjacent points, the neighborhood relationship is shown in Table 1. In each direction it calculates the gray differences between two adjacent points and the center point respectively, if two gray differences are bigger than zero, the corresponding code bit procode $_{k}(i, j)$ of the center point in this direction sets " 1 ", otherwise sets " 0 ". K $(45,90,135,180)$ corresponds the direction respectively.

Table 1. The relation of adjacent points.

\begin{tabular}{|c|c|c|}
\hline $\begin{array}{c}\text { Texture of } 45 \\
\text { degree }\end{array}$ & $\begin{array}{c}\text { Texture of } \\
180 \text { degree }\end{array}$ & $\begin{array}{c}\text { Texture of } \\
135 \text { degree }\end{array}$ \\
\hline $\begin{array}{c}\text { Texture of } 90 \\
\text { degree }\end{array}$ & $\begin{array}{c}\text { Current } \\
\text { center point }\end{array}$ & $\begin{array}{c}\text { Texture of } 90 \\
\text { degree }\end{array}$ \\
\hline $\begin{array}{c}\text { Texture of } \\
135 \text { degree }\end{array}$ & $\begin{array}{c}\text { Texture of } \\
180 \text { degree }\end{array}$ & $\begin{array}{c}\text { Texture of } 45 \\
\text { degree }\end{array}$ \\
\hline
\end{tabular}

Then according to the formula (2) it calculates four directional output values of each basic feature point

$$
\left\{\begin{array}{c}
\operatorname{Direction}(i, j)=(I(i, j-1)+ \\
(i, j+1)-2 * I(i, j)) / 2) \\
\operatorname{Direction}(i, j)=(I(i+1, j-1)+ \\
I(i-1, j+1)-2 * I(i, j)) / 2 \\
\operatorname{Direction}(i, j)=(I(i-1, j-1)+ \\
I(i+1, j+1)-2 * I(i, j)) / 2 \\
\operatorname{Direction}(i, j)=(I(i, j-1)+ \\
(i, j+1)-2 * I(i, j)) / 2)
\end{array}\right.
$$

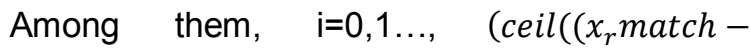
$x_{l}$ match $\left.) / N\right)-1, \mathrm{j}=0,1 \ldots, \quad$ ceil $\left(r_{D}\right.$ match $\left./ M\right)-1$, expresses the corresponding gray value of each basic feature point.

Finally, it eliminates the false feature points, the detail method is as follows: it records the directional number $\mathrm{K}(45,90,135,180)$ with the maximal directional out value of each basic feature point According to formula (3) it makes binary code for each basic feature point, if a directional code of each basic feature point is " 1 " and this directional out value is bigger than three other directional out values, this directional code is still " 1 ", three other directional codes are set " 0 "; otherwise this directional code is set " 0 ". It makes similar operation for four directional codes of each basic feature point.

$$
\begin{aligned}
& \text { Code }(\mathrm{i}, \mathrm{j})= \\
& \left\{\begin{array}{c}
b \quad a=K, \operatorname{procode}(i, j)=b \\
0 \quad a \neq K \quad a=45,90,135,180, b=0,1
\end{array}\right.
\end{aligned}
$$

Among them, a expresses the corresponding directional code bit, so it gets (ceil ( $x_{r}$ match $x_{l}$ match $\left.\left.) / N\right)\right)$ Xceil $\left(r_{D}\right.$ match $\left.\left./ M\right)\right) X 4$ bits.

\section{Iris Matching}

In the collective and effective area, I made match to the entering iris and registering iris. The corresponding codes of the entering iris and registering iris are Register codei, Enroll codei which correspond to the code of each directional output value respectively.

$i=1,2,3,4 ; a=45,90,135,180$.

$i=1$ corresponds to the directional code of 45 degree $\mathrm{i}=2$ corresponds to the directional code of 90 degree $\mathrm{i}=3$ corresponds to the directional code of 135 degree $i=4$ corresponds to the directional code of 180 degree.

When we compare with two iris codes, because the anterior normalized operation cannot solve the revolving invariable problem, 
we need to carry on certain revolving match for registering iris and entering iris. The revolving can becompensated even the corresponding code of the registering iris and the entering iris can not correspond completely. This article solves the revolving invariable problem in the normalized image, this may transform the revolving operation in the annular iris to the translation operation in the rectangular iris. The concrete method is as follows: when it compares with two iris codes, maintains the code of the registering iris motionless, and the code of the entering iris is translated several pixels to left or right along horizontal direction (because the angles of rotation of image is not big, translation pixels are small), it calculates a match value with the registering iris code after translating one pixel, after the translation ends, we keep the maximum of all the match values as the final match value of the registering iris and the entering iris. We sum the match distance by formula (4) as follows:

$\left\{\sum_{K=1}^{K=4 m=\left(\operatorname{ceil}\left(\left(x_{r} \text { match }-x_{l} \text { match }\right) / N\right)\right.}\right.$

$$
\begin{aligned}
M d_{3 \times 3}= & \max _{(p=-2,-1,0,1,2)} \\
& (q=-2,-1,0,1,2)
\end{aligned}
$$

$$
\sum_{m=0}^{n=\operatorname{ceil}\left(r_{D} \text { match } / M\right)} \sum_{n=0}\left[\text { registercode }{ }_{k}(m, n)\right.
$$

Then matching distance is ,

$$
\text { \& } \left.\left.\text { enrollcode }_{k}(m+p, n+q)\right]\right\}
$$

$$
\begin{gathered}
M d=M d_{3 \times 3 /}\left(\operatorname{ceil}\left(\left(x_{r} m a t c h-x_{l} \text { match }\right) / N\right)\right) \\
\left.\mathrm{X} \operatorname{ceil}\left(r_{D} \text { match } / M\right)\right)
\end{gathered}
$$

\section{Experimental Results}

When I carried on the recognition experiment, I weighed the algorithm with false acceptance rate (FAR), false rejection rate (FRR), equal error rate (EER), and correct recognition rate(CRR). Simultaneously I inspected the algorithm with the execution time, including feature extraction time, match time. I used the CASIA in the iris database 567 images, including 81 different irises of eyes, each eye had 7 8-bit images, the resolution is $320^{*} 280$.i carried on the recognition experiments 160461 times, the inter-class experiments was 158760 , the intra-class experiments was 1701. For comparing the infection of size of block, I adopted many sizes of block such as $2^{*} 5,3^{*} 4$, $5^{\star} 5$ and $5^{\star} 7$, I made experiments for above subblock, the distribution of Md is shown in Figure 6.

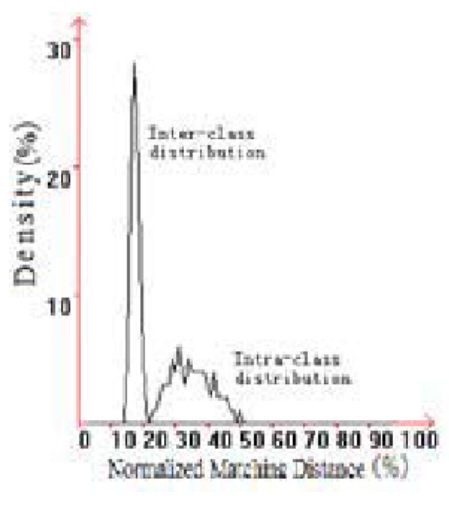

a) Md at $2 * 5$

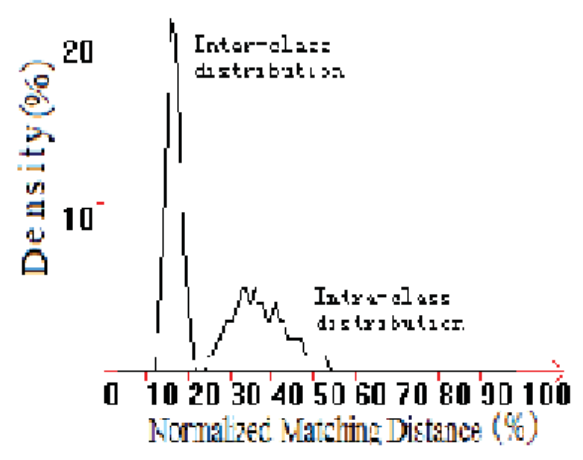

b) Md at $3 * 4$

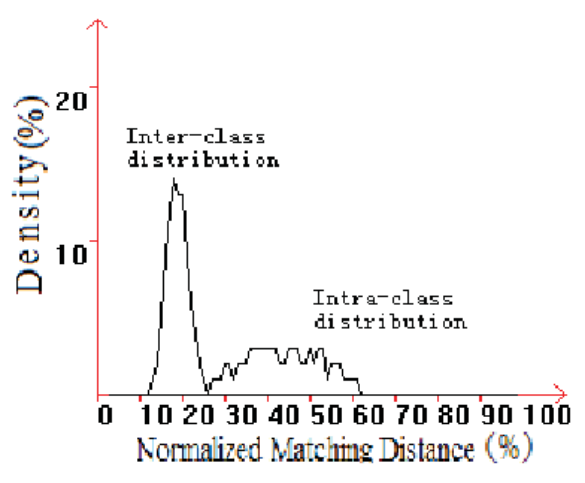

c) Md at $5 * 5$

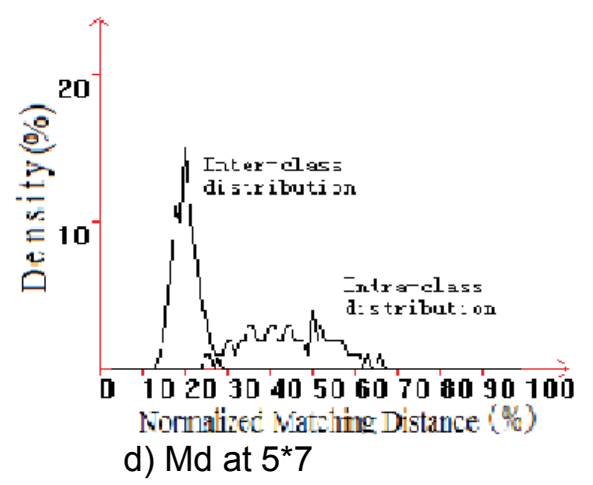

Figure 6. Distribution of Md. 
Table 2. The Accurate Recognition Rate of Different Algorithms

\begin{tabular}{lccccc}
\hline Method & $\begin{array}{c}\text { CRR } \\
(\%)\end{array}$ & $\begin{array}{c}\text { EER } \\
(\mathbf{\%})\end{array}$ & $\begin{array}{c}\text { Feature } \\
\text { extraction } \\
\text { time }(\mathbf{m s})\end{array}$ & $\begin{array}{c}\text { Match } \\
\text { time } \\
(\mathbf{m s})\end{array}$ & $\begin{array}{c}\text { Total time } \\
(\mathbf{m s})\end{array}$ \\
\hline Daugman & 100 & 0.08 & 682.5 & 4.3 & 686.8 \\
Boles & 92.64 & 8.13 & 170.3 & 11 & 181.3 \\
Proposed & 99.687 & 0.303 & 8.3 & 7.2 & 15.5 \\
\hline
\end{tabular}

From Figure 6 we can find that the distribution of the methods of $a$ and $b$ are much better than that of $c$ and d, the crossing area between Interclass distribution and Intra-class distribution of methods $a$ and $b$ are small and it shows the correct recognition rate is high by methods a and $\mathrm{b}$. What is more, the experimental results show when the size of block is too big the correct recognition rate decreases obviously. When the size of block is $3^{*} 4$, the experimental result is best. The threshold of match distance is $0.22922, \mathrm{CRR}=99.687 \%$, FAR $=0.313051 \%$, $\mathrm{FRR}=0.293945 \%$, namely the correct recognition results are 159959 times, the false rejection results are 5 times, the false acceptance results are 497 times. I carried on the duplicated experiments for two previous mentioned methods in the same image samples, the experimental results are listed in table 2 .

The CRR of this article under the threshold value is slightly lower than the Daugman's algorithm, but is higher than Boles's algorithm.

\section{CONCLUSION}

This paper presents a novel iris recognition method based on the natural-open eyes. This method can find the iris characteristic point in a short time, the recognition rate is high, and the recognition speed is guaranteed.

\section{REFERENCES}

Daugman, J. (1993). High confidence visual recognition of persons by a test of statistical independence. IEEE Transactions on Pattern Analysis and Machine Intelligence 15(1): 1148-1161.
Boles, W., Boashash, B. (1998). A human identification technique using images of the iris and wavelet transform. IEEE Transaction on Signal Processing 46(4): 1185-1188.

Wildes, R. (1997). Iris recognition: an emerging biometric technology. Proceedings of IEEE 85(9): 1348-1363.

Institute of Automation, Chinese Academy of Sciences. CASIA Iris Image Database (ver1.0) [DB]. http://www.sinobiometrics.com. 2004. 